\title{
Prenatal Vitamin
}

National Cancer Institute

\section{Source}

National Cancer Institute. Prenatal Vitamin. NCI Thesaurus. Code C92860.

A vitamin enriched supplement containing higher doses for specific nutrients such as folic acid, intended for women before, during and after pregnancy and also during their lactating phase. 